\title{
DIAGNÓSTICO DE TRANSFORMADORES MEDIANTE ANÁLISIS DE RESPUESTA POR BARRIDO DE FRECUENCIA (SFRA)
}

Sáenz Pérez, J.M. ${ }^{1}$; Encina Jiménez, Carlos M. ${ }^{1}$; Piccoli, Gonzalo F. ${ }^{1}$

\section{INTRODUCCIÓN}

El método de análisis de respuesta por barrido de frecuencia SFRA (SweepFrequency Response Analysis), es una técnica de diagnóstico para detectar deformaciones y desplazamientos (entre otras fallas eléctricas y mecánicas) sobre los devanados en transformadores. La detección del problema se traduce directamente en el tipo de mantenimiento que se debe realizar.

Las ventajas principales del SFRA, son su sensibilidad a las distintas fallas que se pueden presentar en arrollamientos y obtención del estado del transformador de manera no invasiva, permitiendo la evaluación de la integridad de los transformadores de potencia sin aplicar altas tensiones, reduciendo costos y tiempo.

El objetivo es conocer la situación de transformadores que han sido sometidos a esfuerzos electrodinámicos, con funcionamiento anómalos, con fallas no detectables por métodos tradicionales (aislación, relación de transformación, corriente vacío) para proceder a su reparación previa a su posible destrucción por cortocircuito interno. Interpretar y aplicar los nuevos resultados de las curvas de respuesta en frecuencia de la función de transferencia del transformador, con el uso de este nuevo ensayo.

Para ello se introducen algunas definiciones pertinentes:

\section{DEFORMACIÓN DE DEVANADO:} Este término encierra los cambios de dimensión axial o radial de las bobinas o devanados del transformador de potencia, debido a los esfuerzos mecánicos y electrodinámicos, que generalmente se muestra como una distorsión parcial, abultamiento o el desplazamiento de la bobina, etcétera. La deformación de bobinados puede ocurrir en caso de que el transformador se someta a cortocircuito, a corriente de inserción o sufra golpes durante el transporte, lo que afectara directamente a la operación segura del mismo.

FUNCIÓN DE TRANSFERENCIA $\mathrm{H}(\mathrm{j} \omega)$ : Es la relación entre la señal de salida y la señal de entrada del circuito equivalente del transformador, mostrada en la forma de la transformada de Lapla-

\footnotetext{
${ }^{1}$ Departamento de Ingeniería - Cátedra de Electrotecnia II - FACENA -UNNE -Av. Libertad N 5600, Ctes. Capital. Ing. Sáenz Pérez: (0379) 154606687 - jmsaenzperez@yahoo.com.ar Carlos M. Encina Jiménez: (03794)15298146 - martinencina18@gmail.com Gonzalo F. Piccoli: (03482) 15230404 - gonzapiccoli@gmail.com
} 
ce. La distribución de polos y ceros de la función de transferencia está estrechamente relacionada con los parámetros internos, el método de conexión y la impedancia de los terminales de conexión de la red bilateral.

$$
H(j \omega)=\frac{\text { Señalsalida }(j \omega)}{\text { Señaldeentrada }(j \omega)}
$$

\section{RESPUESTA DE FRECUENCIA:} Es la relación ente $H(j \omega)$ (función de transferencia) y $\omega$ (frecuencia angular de la red) cuando se alimenta al circuito con una señal senoidal constante en amplitud. La variación de la amplitud de $\mathrm{H}(\mathrm{j} \omega)$ en función de $\omega$ es generalmente denominada como respuesta en frecuencia de amplitud, mientras que la variación de la fase de $\mathrm{H}(\mathrm{j} \omega)$ en función de $\omega$ se conoce como fase de la respuesta de frecuencia.

\section{ANÁLISIS DE LA RESPUESTA DE} FRECUENCIA (FRA): Se define análisis de respuesta en frecuencia a cualquier medida de la respuesta eléctrica de los bobinados del transformador (función de transferencia) en función de la frecuencia. Por medio de esta medida se pretende determinar cualquier deformación de las bobinas a través de los efectos de los cambios resultantes en la distribución de capacidades o inductancias.

\section{MÉTODO DE BARRIDO DE FRE-} CUENCIA: Se denomina así al proceso por el cual se mide la respuesta en frecuencia del circuito equivalente cuando se inyecta una señal de frecuencia variable.

\section{FRECUENCIA DE RESONAN-} CIA: Las frecuencias correspondientes a los máximos o mínimos locales en la respuesta de la amplitud medida.

\section{Análisis de la respuesta de barrido de frecuencia (SFRA)}

La medición de la respuesta en frecuencia se fundamenta en el concepto de que las variaciones debidas a deformación y desplazamiento de los devanados en el transformador se reflejan en un cambio de los parámetros L, C, y R del circuito equivalente del devanado, modificando así su respuesta en frecuencia.

Por definición general el SFRA consiste en realizar la medición en los devanados del transformador de la magnitud y la fase, ya sea de la Impedancia o Función de transferencia, cuando se les aplica una señal sinusoidal en una banda amplia de frecuencias, comparando luego las mediciones con un conjunto de registros de referencia, pudiéndose tratar de registros del mismo transformador (mediciones homólogas), de registros de otro transformador con igual diseño, o también de registros pertenecientes a las otras fases del mismo transformador (mediciones Inter-Fases).

Esta definición general ha dado paso a que aspectos relacionados con la metodología SFRA y el diagnóstico mismo no hayan sido unificados. Por lo que buscamos unos resultados generales, que permitan identificar una posible falla mecánica en el transformador.

Los problemas mecánicos internos que se pueden encontrar en el transformador, son:

- Movimiento del núcleo.

- Deformación y desplazamiento del arrollamiento.

- $\quad$ Fallas en el núcleo.

- Colapso parcial del arrollamiento.

- Estructuras de sujeción rotas y/o 
sueltas.

- Cortocircuitos en arrollamientos.

- Daños que haya experimentado el transformador después de terremotos, estrés mecánico, transporte, rayos u otros factores ambientales.

\section{Metodología del SFRA}

Se inyecta una señal de baja amplitud a cada extremo del bobinado de un transformador y medir en el extremo opuesto la atenuación que dicho bobinado produce. La amplitud de la señal se mantiene constante durante todo el ensayo y se varia la frecuencia de la misma para detectar los máximos y mínimos de la tensión de sali$\mathrm{da}$, se calculan las atenuaciones y se vuelcan en un gráfico atenuación versus frecuencia o se introduce los datos obtenidos en un programa de computadora que realiza los cálculos y grafica los resultados en la escala que se desee, permitiendo obtener una idea del estado interno del transformador antes del desencubado del mismo, la relación entre estas señales de entrada y salida provee la respuesta de frecuencia o función de transferencia del transformador. El modelo equivalente posee una impedancia diferente en cada frecuencia, entonces ante cualquier deformación en la geometría del sistema cambia el circuito RLC, y a su vez su respuesta en frecuencias. El instrumental básico utilizado para la medición está conformado por un generador de barrido, un detector de dos canales y un registrador.

\section{Procedimiento de medición}

El SFRA es una prueba que se realiza con en el equipo desenergizado, es recomendable blindar en forma coaxil los cables y puntas de prueba para minimizar los efectos de capacidad distribuida. Existen diferentes tipos de prueba para realizar la medición:

\section{Admitancia de Circuito Abierto (CA)}

Se realiza entre los extremos o terminales del mismo devanado, con todos los demás terminales flotantes. La impedancia de magnetización del transformador es el principal parámetro que caracteriza la respuesta de baja frecuencia en esta configuración (bajo la primer resonancia). Se la utiliza por su simplicidad y facilidad de analizar cada devanado por separado.

\section{Admitancia de cortocircuito (CC)}

Se realiza entre los extremos o terminales de un mismo devanado, mientras el devanado de baja tensión es cortocircuitado.

La influencia del núcleo desaparece por debajo de aproximadamente $10-20 \mathrm{kHz}$ porque la respuesta de baja frecuencia se caracteriza por la impedancia de cortocircuito/reactancia de fuga en lugar de la inductancia de magnetización, la respuesta en alta frecuencia crea caminos de flujo de dispersión que puede causar modificaciones en la información directamente relacionada al devanado bajo prueba.

Debido a que en altas frecuencias, la prueba en cortocircuito crea caminos de flujo de dispersión, se puede complementar con la prueba de circuito abierto, para frecuencias superiores a $20 \mathrm{kHz}$.

\section{Aplicación del SFRA}


La respuesta en frecuencia de cada modelo es única, y se la puede considerar como una "huella dactilar" de cada transformador.

Existen dos categorías distintas para la aplicación de medidas de respuesta en frecuencia, en la fábrica y en el campo. En ambos casos, los procedimientos y precauciones para generar una buena medida son los mismos. Sin embargo, hay una diferencia en la motivación de las pruebas en cada categoría.

Aplicación en la fábrica: Hay dos razones para generar medidas de respuesta en frecuencia dentro de un ambiente de fábrica:

- Garantía de calidad y;
- Base de referencia antes del transporte.

Aplicación en el campo: Las dos razones que generan la necesidad de realizar el ensayo en campo son:

- Reubicación de la unidad (después del transporte desde fábrica o relocalización de la unidad) y;

- Luego de un incidente (rayos, fallas eléctricas, cortocircuito, un evento sísmico, etc.).

\section{Circuito equivalente de un transforma- dor:}

Se puede modelar al transformador, teniendo en cuenta los acoples capacitivos e inductivos, como se aprecia en la figura:
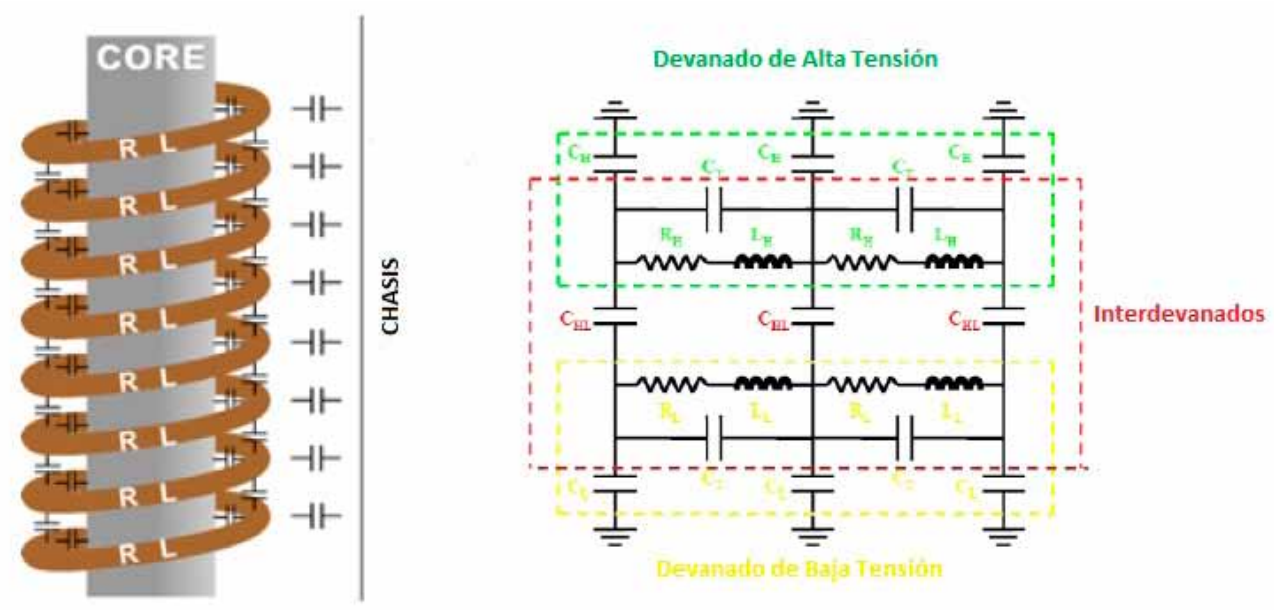

Fig. 1-Modelo equivalente del transformador 


\section{Normativa de FRA:}

Las normas que regulan los ensayos de respuesta en frecuencia son: IEEE C 57.149, IEC 60076-18, CIGRE 342_2008. Estas normas determinan los rangos de frecuencias en las que se puede determinar el tipo de falla correspondiente a cada transformador, estas se resumen en la siguiente gráfica:

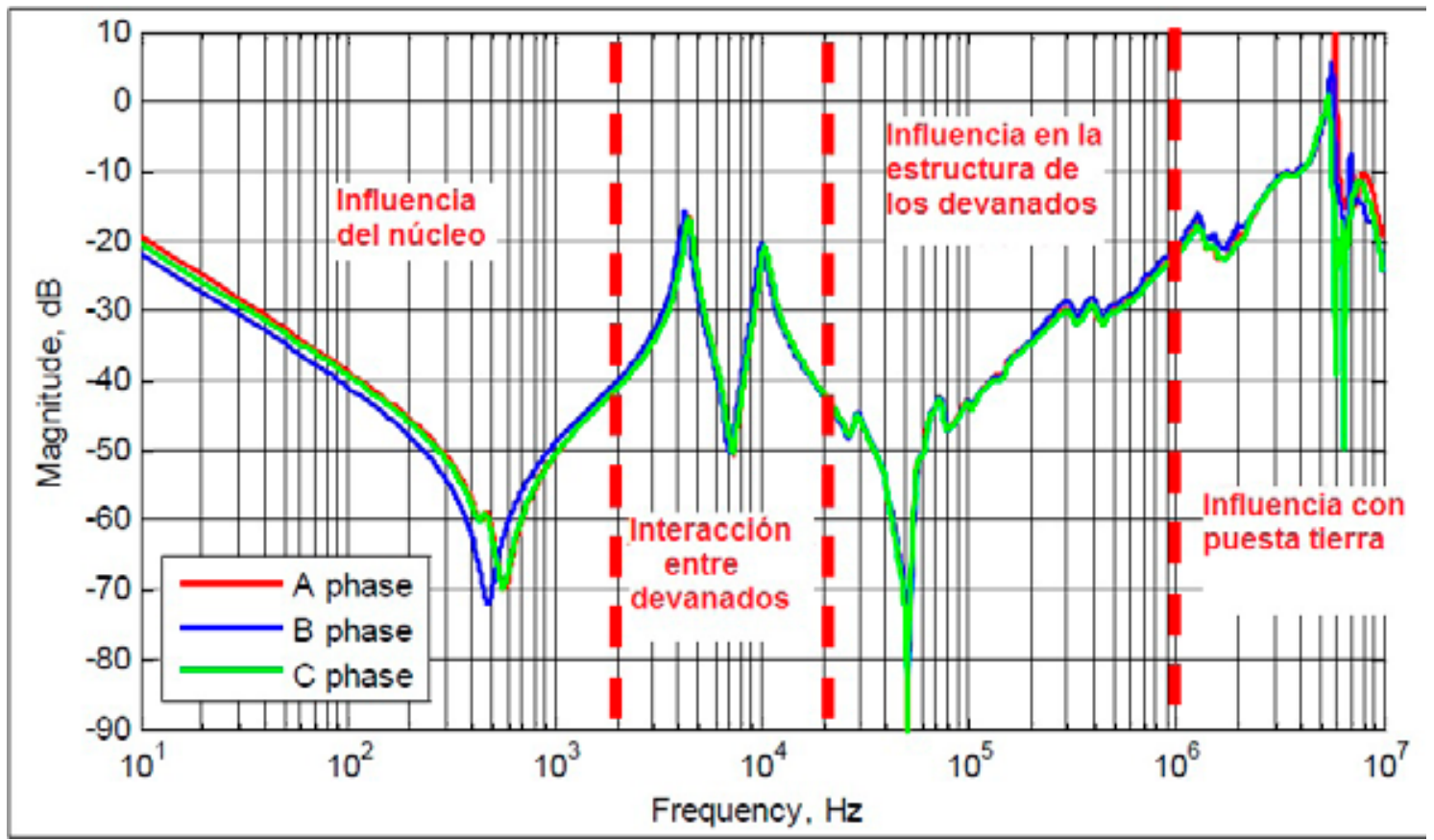

Fig. 2-Regiones de frecuencia según Norma IEEE

En general, las fallas encontradas en un transformador según la frecuencia aplicada son:

\section{Bajas frecuencias:}

- Problemas en el núcleo.

- Devanados abiertos / cortocircuitados.

- Malas conexiones.

- Cambios en la impedancia de cortocircuito.

Frecuencias medias:

- Deformaciones en los devanados.
- Desplazamiento de los devanados.

Altas frecuencias:

- Movimiento de los devanados y conexionado de conmutador.

\section{Análisis de resultados}

Utilizando el simulador PSIM, se realizó 


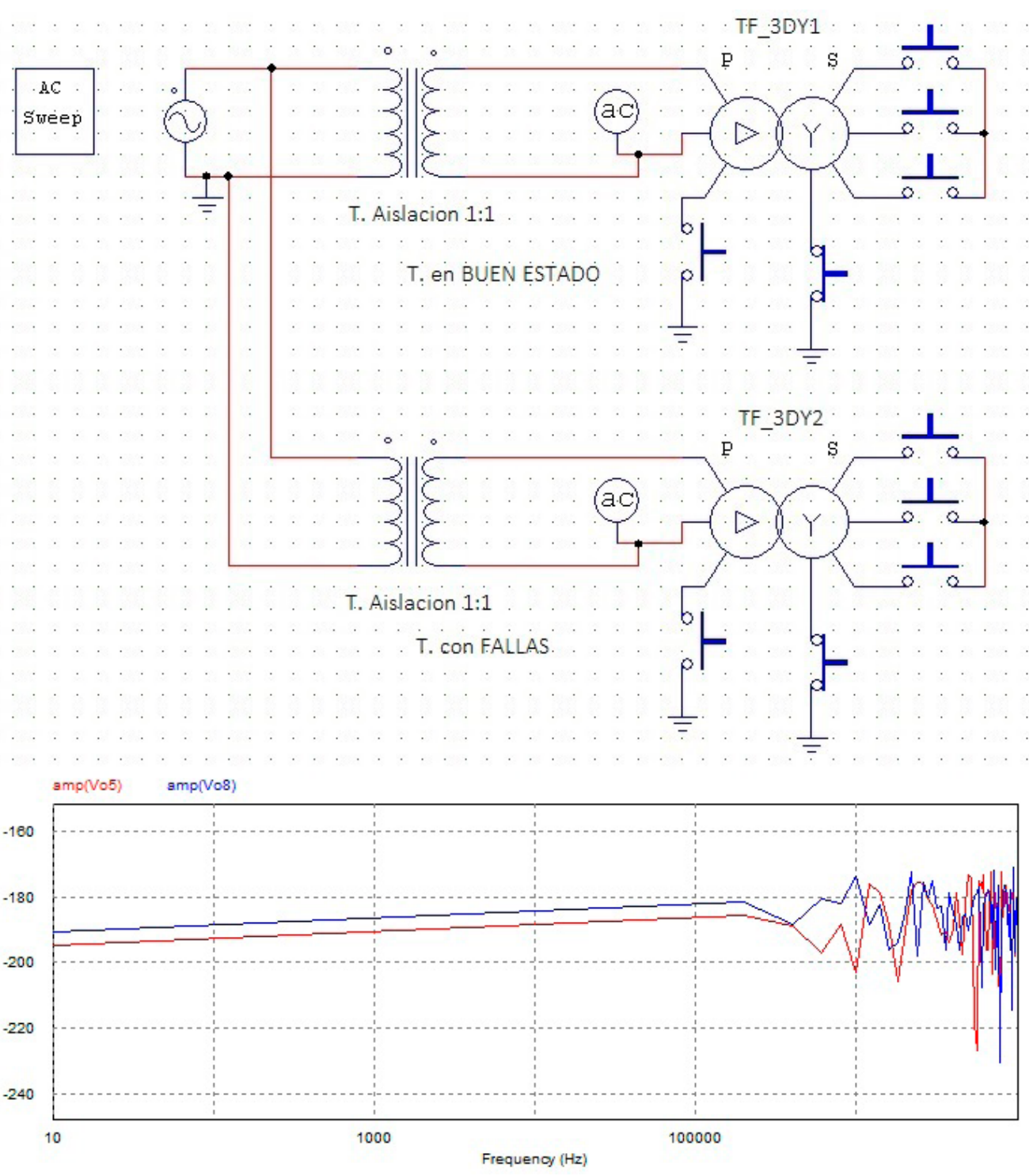

Fig. 3- Ensayo en PSIM

la prueba de respuesta en frecuencia:

E1 transformador TF_3DY1, es un transformador trifásico DY que se utilizó como referencia, es decir en buen estado (Vo8). Para realizar el barrido de frecuencias se utilizó la función AC SWEEP, de 10 $\mathrm{Hz}$ a $1 \mathrm{MHz}$. Al transformador TF_3DY2, se lo modificó para simular fallas en el nú- cleo y en los devanados, comparando las respuestas de ambos transformadores, en azul el transformador en buen estado.

Se observa una desviación en la zona de bajas frecuencias que es indicativo de cambios en el circuito magnético, defectos del núcleo. Además se observa grandes desviaciones en media y alta frecuencia, que son 
indicativos de fallas en los devanados, estos resultados advierten la necesidad de reparaciones de la unidad.

\section{Conclusión SFRA}

Es un método comparativo para evaluar el estado de los transformadores de poten- cia. Para evaluar los resultados de FRA, los datos se comparan con los datos de referencia, ya sea por inspección visual directa de las curvas o mediante el uso de los datos procesados. Esto, teniendo en cuenta la siguiente tabla que resume las fallas según la frecuencia aplicada:

\begin{tabular}{|c|c|}
\hline $\begin{array}{c}\text { Comparación entre análisis futuros y } \\
\text { análisis base }\end{array}$ & Falla o diagnóstico \\
\hline $\begin{array}{l}\text { Cambios en la forma de la traza o } \\
\text { distorsión en la misma, para frecuencias } \\
\text { menores a } 5 \mathrm{kHz}\end{array}$ & $\begin{array}{l}\text { Problemas del Núcleo, debido a golpes o } \\
\text { cambios en sus propiedades magnéticas. }\end{array}$ \\
\hline $\begin{array}{l}\text { Cambios en la forma de la traza o } \\
\text { distorsión, para frecuencias mayores a } \\
10 \mathrm{kHz}\end{array}$ & Problemas de los devanados, \\
\hline $\begin{array}{l}\text { Cambios menores a } 3 \mathrm{~dB} \text { respecto a la } \\
\text { traza base. }\end{array}$ & $\begin{array}{l}\text { Transformador en estado normal, las } \\
\text { pequeñas variaciones se deben a } \\
\text { tolerancias. }\end{array}$ \\
\hline $\begin{array}{l}\text { Cambios de }+/-3 \mathrm{~dB} \text { o más, entre } 5 \mathrm{~Hz} \text { y } \\
2 \mathrm{Khz}\end{array}$ & $\begin{array}{l}\text { Cortocircuito de espiras, desplazamiento } \\
\text { del núcleo }\end{array}$ \\
\hline $\begin{array}{l}\text { Cambios de }+/-3 \mathrm{~dB} \text { o más, entre } 50 \mathrm{~Hz} \text { y } \\
20 \mathrm{Khz}\end{array}$ & Desplazamiento de los devanados. \\
\hline $\begin{array}{l}\text { Cambios de }+/-3 \mathrm{~dB} \text { o más, entre } 500 \mathrm{~Hz} \text { y } \\
2 \mathrm{Mhz}\end{array}$ & Deformación en los devanados \\
\hline $\begin{array}{l}\text { Cambios de }+/-3 \mathrm{~dB} \text { o más, entre } 25 \mathrm{~Hz} \text { y } \\
10 \mathrm{Mhz}\end{array}$ & $\begin{array}{l}\text { Problemas en los bornes del } \\
\text { transformador } y / o \text { puntas de prueba mal } \\
\text { conectadas }\end{array}$ \\
\hline
\end{tabular}

Tabla 1- Resumen de identificación de fallas.

Las normativas en la Argentina no se encuentra normalizado para el análisis de respuesta en frecuencias, por lo que esperamos contribuir a la normativa con este trabajo. La investigación sobre el ensayo continua, buscando un modelo circuital más relevante del transformador. Se inició el diseño de un dispositivo para realizar el SFRA de manera automática, utilizando para ello un oscilador controlado por tensión MAX038, que genera la onda senoidal. 


\section{Bibliografía}

1. Melo, Leonardo. (2012). Aplicación de Técnicas Estadísticas al Ensayo de Análisis de Respuesta en Frecuencia en Transformadores de Potencia.

2. J. Secue, E. Mombello, SeniorMember, IEEE y C. V. Cardoso. (2007). Revisión del Análisis de Respuesta en Frecuencia (SFRA) para Evaluación de Desplazamientos y Deformaciones de Devanados en Transformadores de Potencia.
3. ShivangiRai, (Prof.) N.P. Gupta. (2014). SFRA, Detect Of Winding Deformation in Power Transformer.

4. Ing. Oghievski Alejandro. (1984). Ditección del Desplazamiento de los Bobinados en un Transformador, debido a los esfuerzos electrodinámicos de cortocircuitos.

5. Néstor Xavier Maya Izurieta y Luis Alberto Vásquez Restrepo. (2011). Pruebas de transformadores, espectroscopia en el dominio de la frecuencia y método de análisis de respuesta por barrido de frecuencia. 